\title{
Energy exchange via multi-species streaming in laser-driven ion acceleration
}

\author{
M. King ${ }^{1}$, R. J. Gray ${ }^{1}$, H. W. Powell ${ }^{1}$, R. Capdessus ${ }^{1}$ and \\ P. McKenna ${ }^{1}$ \\ ${ }^{1}$ SUPA Department of Physics, University of Strathclyde, Glasgow G4 0NG, \\ UK \\ E-mail: paul.mckenna@strath.ac.uk
}

\begin{abstract}
Due to the complex electron dynamics and multiple ion acceleration mechanisms that can take place in the interaction of an ultra-intense laser pulse with a thin foil, it is possible for multiple charged particle populations to overlap in space with varying momentum distributions. In certain scenarios this can drive streaming instabilities such as the relativistic Buneman instability and the ion-ion acoustic instability. The potential for such instabilities to occur are demonstrated using particle-in-cell simulations. It is shown that if a population of ions can be accelerated such that it can propagate through other slowly expanding ion populations, energy exchange can occur via the ion-ion acoustic instability.
\end{abstract}

PACS numbers: 41.75.Jv, 52.38.-r, 52.38.Kd 


\section{Introduction}

Ultra-intense $\left(>10^{20} \mathrm{Wcm}^{-2}\right)$ laser radiation interacting with solid density targets results in strong electric fields that can be used to accelerate ions to multi-MeV energies over micron-scale distances [1]. This can produce high energy ion beams that have the potential to be utilised in diverse applications such as oncology [2], radiography [3] and fast-ignition inertial confinement fusion [4]. It is important to understand the underlying physical processes that may occur during the various ion acceleration mechanisms in order to enhance and control the energies and other properties of the resultant ion beams.

There are a variety of acceleration mechanisms that can occur when a sub-micron-thick foil target is irradiated by an ultra-intense laser pulse. Target normal sheath acceleration (TNSA) is the most studied mechanism and occurs due to the formation of a strong electrostatic field $(\sim \mathrm{TV} / \mathrm{m})$ on the rear surface, due to the propagation of fast electrons through the target from the laser-irradiated front side [5]. This tends to produce ion beams that are divergent with a thermal energy spectrum and maximum energy which scales with the electron temperature (i.e. the square root of the laser intensity in the relativistic regime) $[6,7]$. The various ion species produced become separated according to their charge-to-mass ratio [8]. A more promising acceleration mechanism is that of radiation pressure acceleration (RPA) in which the front surface of the target is driven forward by the laser radiation pressure [9]. This has been theorised to produce ion beams with low divergence, narrow peaked energy spectra and a more favourable energy scaling with laser intensity $[10,11]$.

If the heated target electron population expands sufficiently during the laser pulse interaction, such that the peak target density decreases below the relativistically-corrected critical density, $n_{c}^{\prime}, \quad\left(n_{c}^{\prime}=\right.$ $\gamma m_{e} \epsilon_{0} \omega_{L}^{2} / e^{2}$, where $m_{e}$ is the electron rest mass, $\epsilon_{0}$ is the vacuum permittivity, $\omega_{L}$ is the angular laser frequency, $e$ is the electron charge and $\gamma$ is the Lorentz factor), then relativistic induced transparency (RIT) occurs. Transparency can also occur in ultrathin foils by compression of the electron density to a thickness less than the skin depth [12]. The remainder of the laser pulse propagates through the target, heating the electrons throughout the volume, which can result in the production of electron beams with controllable mode structures $[13,14]$ and high energy electron jets [15]. These electrons can stream with the expanding ions at the rear of the target, facilitating energy exchange with the ions. This exchange of energy has been suggested to be due to the relativistic Buneman instability $[16,17]$ and is the basis of the ion acceleration mechanism known as breakout afterburner (BOA) $[18,19]$. Finally, in thin foils which expand to the extent that the laser propagates through an extended near-critical density plasma, ions can be accelerated by the formation of magnetic vortices in the decreasing density gradient $[20,21]$ or via collisionless shock wave acceleration.

The case of an initially solid density foil that becomes relativistically transparent during the laser pulse interaction is particularly interesting because of the various particle populations that can stream through the same region of space. These include TNSA-ions from the rear surface, fast electrons pondermotively accelerated when the laser pulse propagates through the target volume and RPAions from the front or bulk of the target as the laser pulse bores into it (the process of hole-boring). Depending upon the density and relative drift of the different particle streams, it is possible for electrostatic, longitudinal instabilities to grow and facilitate energy exchange between the particle populations.

In this article, we explore the potential for energy exchange via the streaming of multiple ion and electron populations as a thin foil target is irradiated by an ultra-intense laser pulse. Signatures of the effects of streaming are observed in 1D particle-in-cell (PIC) simulations of an ultraintense laser pulse interacting with a pre-expanded thin foil that undergoes RIT. Whilst the acceleration mechanisms that lead to the streaming behaviour are found to be much less efficient in simulations with a higher dimensionality, the underlying principle of energy exchange via streaming can still hold true. Tailored 2D simulations, removing the effect of the laser pulse, show that if the same streaming behaviour occurs, the energy exchange mechanism observed in the 1D simulations remains the same. The unstable wave growth that occurs in these simulations can be shown to be comparable with that of the established linear theory for Buneman [16] and ion-ion acoustic instabilities [22]. 


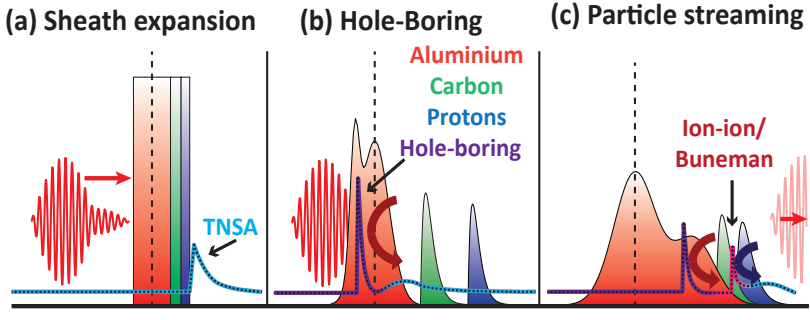

Figure 1. Schematic illustrating the three stages of the forwarddirected ions of the 1D laser-acceleration simulation: (a) TNSA is produced early in the simulation; (b) The radiation pressure on the rising edge of the pulse results in hole-boring into the target; (c) The target becomes relativistically transparent leading to streaming between fast, volumetrically heated electrons, TNSAion layers and the faster hole-boring accelerated ions.

\section{One-dimensional laser-solid simulations}

To investigate the potential role of the relativistic Buneman and ion-ion acoustic instabilities in laser-foil interactions, 1D PIC simulations have been performed utilising the EPOCH code [25]. These simulations involve a $1.054 \mu \mathrm{m}$ wavelength laser pulse interacting with an initially solid density target foil with a hydrocarbon contamination layer on both the front and rear surfaces, initialised as illustrated in Fig. 1(a). A similar scenario was previously investigated experimentally by our group and the results are reported in Powell et al [15]. The target is initialised as a $40 \mathrm{~nm}$-thick $\mathrm{Al}^{11+}$ slab with a solid density $\left(60 n_{c}\right)$ with $8 \mathrm{~nm}$-thick contamination layers of mixed hydrocarbon $\left(\mathrm{C}_{2} \mathrm{H}_{6}\right)$ comprising $\mathrm{C}^{6+}\left(20 n_{c}\right)$ and $\mathrm{H}^{+}$ $\left(60 n_{c}\right)$ ions. Electrons are initialised to neutralise the ion charge, with a starting temperature of $1 \mathrm{keV}$. The simulation box was $310 \mu \mathrm{m}$ in length with 381,540 simulation mesh cells giving a mesh cell size of $0.81 \mathrm{~nm}$. The laser pulse intensity is defined as $2 \times 10^{20} \mathrm{Wcm}^{-2}$ with a pre-pulse at the $10 \%$ level (with a peak-topeak separation of $1.5 \mathrm{ps}$ ) to initialise electron heating and pre-expansion of the target. The laser enters the simulation box from the left-hand boundary edge, 10 $\mu \mathrm{m}$ from the target surface, with the peak of the initial pulse entering at a simulation time of $0.8 \mathrm{ps}$. This drives a hole-boring front as illustrated schematically in Fig. 1(b), resulting in a fast population of ions and electrons which interact with the TNSA expanded ions giving rise to streaming instabilities (Fig. 1(c)). Even without a pre-pulse, streaming occurs but closer to the original target surface. The pre-pulse enables non-idealised laser drive parameters to be achieved and the streaming to be investigated. A dampening factor was included to reduce the laser pulse intensity with distance in accordance with an expanding Gaussian beam with a minimum waist of $3.5 \mu \mathrm{m}$ at the target surface.

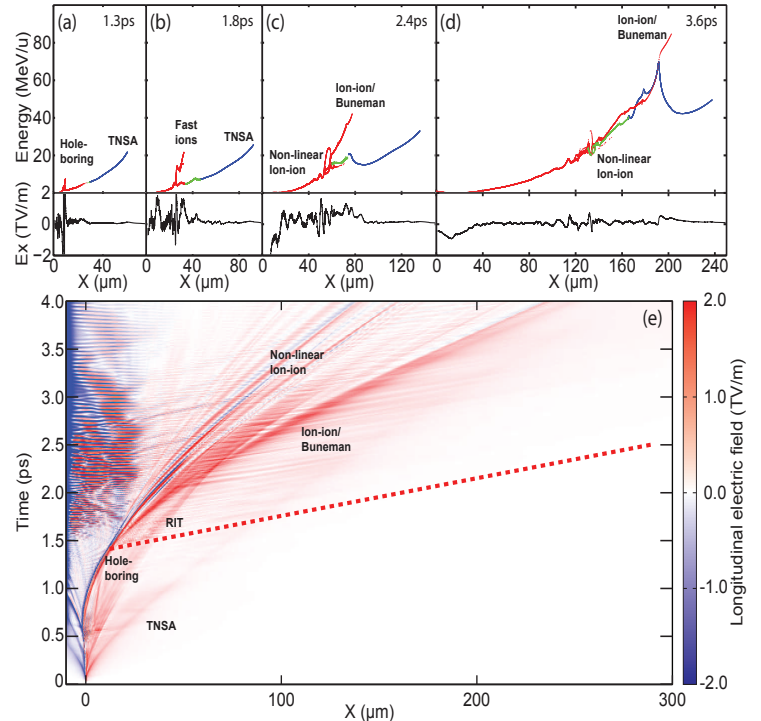

Figure 2. 1D simulation results: (a)-(d) energy-space plots at 4 given time-steps with the corresponding longitudinal electric field below (black). Red - $\mathrm{Al}^{11+}$ ions: green $-\mathrm{C}^{6+}$ ions and blue protons. (e) Time and space evolution of the longitudinal electric field. The dashed line indicates the leading edge propagation of the laser pulse after the onset of transparency

As the pre-pulse interacts with the target, the target electron population is heated and starts to expand. Fast electrons are driven forward through the target. This produces strong electrostatic longitudinal fields due to space-charge separation. TNSA occurs, as observed in the ion phase-space plot shown in Fig. 2 (a). As the laser pulse intensity increases, the peak target electron density reduces due to the expansion and additional heating.

As the target density (still overdense) approaches the relativistic critical density, the laser pressure begins to drive the front surface of the target forwards, resulting in hole-boring. Fig. 2(a) also shows the formation of the hole-boring front in the aluminium phase-space and the associated electrostatic field. This front accelerates a small proportion of the heavy aluminium ions to velocities greater than that of the expanding TNSA-ion population at the rear surface, Fig. 2(b). As this fast component of the aluminium ions propagates through the layers of slower carbon ions and protons, the ion-ion acoustic instability grows as shown in Fig. 2(c) resulting in energy exchange between the species. As the streaming occurs between the $\mathrm{Al}^{11+}$ and $\mathrm{H}^{+}$ions, a density perturbation in both species begins to grow giving rise to a growing longitudinal electric field. This results in the predominant deceleration of the fast $\mathrm{Al}^{11+}$ population on one side of the field and acceleration of the slow $\mathrm{H}^{+}$population on the other. Due to the lower charge-to-mass ratio of the $\mathrm{Al}^{11+}$ ions, this field 

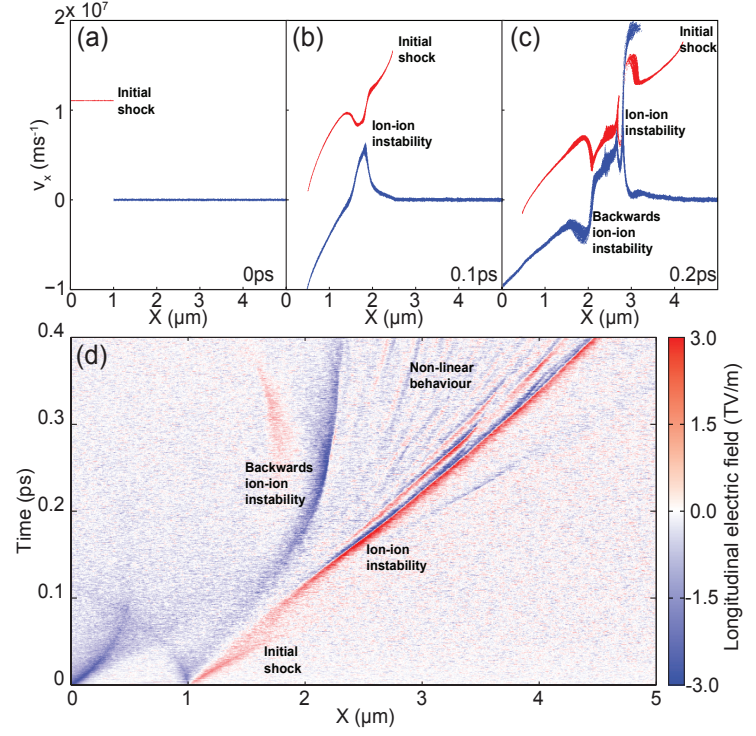

Figure 3. (a)-(c) Phase-space plots from a 2D simulation showing the instantaneous velocities of the $\mathrm{Al}^{11+}$ ions (in red) and protons (in blue) at $0 \mathrm{ps}, 0.1 \mathrm{ps}$ and $0.2 \mathrm{ps}$. (d) Time-space evolution of the longitudinal electric field along the central axis of the $2 \mathrm{D}$ simulation.

affects the $\mathrm{H}^{+}$population more readily, leading to more effective acceleration of the $\mathrm{H}^{+}$population, as shown in Fig. 2(d).

In thin targets which become relativistically transparent during the interaction, the remainder of the laser pulse then propagates through the target, directly heating the electron population within the target volume and at the rear. This can give an additional boost to the ions through the space-charge separation of the electron and ion populations as well as a potential energy coupling between the ions and the electrons via the Buneman instability as discussed by Albright et al.[17].

Fig. 2(e) shows the temporal and spatial behaviour of the longitudinal electric field throughout the simulation. The initial strong field component before transparency occurs corresponds to that of the hole-boring front. After transparency, the electric field propagates forward at a much faster rate and grows corresponding to the fast $\mathrm{Al}^{11+}$ population streaming into the TNSA-proton layer, in addition to the field growth corresponding space-charge separation and potentially the Buneman instability. This corresponds to one example scenario in which the streaming of charged particles with different relative energies can occur in laser-foil interactions and can potentially result in energy exchange.

\section{Two-dimensional ion-ion streaming simulations}

It is found that when the same initial conditions for the 1D simulations are applied to 2D simulations, the $\mathrm{Al}^{11+}$ velocities produced are much lower and do not reach the expanding proton layer. This prevents a direct comparison of the streaming behaviour in $1 \mathrm{D}$ and $2 \mathrm{D}$ scenarios. Instead 2D simulations are initialised with the resultant density and velocity components obtained from the 1D simulation after the $\mathrm{Al}^{11+}$ ions are accelerated. In such a scenario, the laser pulse is no longer present, enabling both the verification of the streaming behaviour observed in the 1D simulations and evidence that the same behaviour can occur without the presence of the laser pulse.

At the point where streaming begins to occur in the 1D simulations, the electron temperature increases to the range $\sim 0.2-2 \mathrm{MeV}$ due to direct heating by the laser. The density of the fast $\mathrm{Al}^{11+}$ component is an approximately uniform bunch of $\sim 0.01 n_{c}$ whilst the $\mathrm{H}^{+}$density is $\sim 0.1 n_{c}$ at the rear surface and decreases exponentially in the positive longitudinal $(+\mathrm{X})$ direction due to TNSA driven expansion. As the majority of the streaming interaction between the two species occurs over a relatively small spatial extent in the $\mathrm{X}$-axis at the rear of the $\mathrm{H}^{+}$layer, the $2 \mathrm{D}$ simulations can be simplified with a uniform longitudinal density. The 2D simulations were therefore initialised to consist of a uniform density $\mathrm{Al}^{11+}$ slab propagating into a uniform $\mathrm{H}^{+}$slab in the presence of a neutralising, hot electron population. The density of the $\mathrm{Al}^{11+}$ species was chosen to approximate that of the $1 \mathrm{D}$ simulation fast population of $0.01 n_{c}$. The proton density was chosen to be 11 times higher to ensure an isotropic electron population density across both ion populations to minimise electrostatic shock formation from electron density discontinuities [26]. Whilst this is idealised, the $\mathrm{H}^{+}$to fast $\mathrm{Al}^{11+}$ density ratio from the $1 \mathrm{D}$ simulations is comparable. As it is only the relative drift velocities between the populations that is important, the proton and electron drift velocities were set to zero whilst the $\mathrm{Al}^{11+}$ drift was $1.2 \times 10^{7} \mathrm{~ms}^{-1}$, again corresponding to $1 \mathrm{D}$ simulation results. The electron temperature was set to $0.5 \mathrm{MeV}$ with both ion species temperatures at $100 \mathrm{eV}$. The simulation box was $30 \mu \mathrm{m} \times 28.8 \mu \mathrm{m}$ with $5000 \times 2880$ mesh cells.

Fig. 3 shows the phase-space plot of the two ion species from 0 to 0.2 ps along the longitudinal dimension for a transverse window of $2 \mu \mathrm{m}$ width. It is clear that energy exchange is occurring as the $\mathrm{Al}^{11+}$ ions propagate through the proton layer which can be compared to the $1 \mathrm{D}$ simulation phase-space plots shown in Fig. 2(d) after transparency has occurred. In this scenario, a secondary instability 
is also formed due to the thermal expansion of the proton slab, i.e. the backward propagating protons are counter-streaming with the $\mathrm{Al}^{11+}$ ions. Fig. 3 shows the temporal behaviour of the longitudinal field along the central axis of the simulation. At the beginning of the simulation there is an initial shock induced due to the positive space-charge build up due to the movement of the $\mathrm{Al}^{11+}$ ions into the $\mathrm{H}^{+}$ions [27]. This dissipates after $0.05 \mathrm{ps}$ as the electrons move to compensate for the induced current flow. This initial shock serves to create a density perturbation in the system and provides a starting acceleration of a population of protons. As the $\mathrm{Al}^{11+}$ ions continue to propagate, a separate electrostatic field begins to grow between these and the accelerated protons, reaching a maximum by $0.2 \mathrm{ps}$. This maximum field strength corresponds to the ion-ion acoustic instability achieving saturation (observed in the phase-space vortex formed in Fig 3(c)). The backwards wave can be also be observed growing with the inverse polarity and becomes almost stationary in space after $0.2 \mathrm{ps}$ due to the counter-streaming nature of the ion populations. After saturation, additional electrostatic fields can be seen to grow due to the non-linearities induced by the saturation of the instability (resulting from the addition of multiple streaming populations).

\section{Dispersion relations}

When considering streams of particles, it is possible to derive a $1 \mathrm{D}$, general dispersion relation for an arbitrary number of streams of infinite length from the cold fluid equations [23]. This is given as:

$\sum_{s} \frac{\omega_{p s}^{2}}{\left(\omega-k v_{s}\right)^{2}}=1$

where $\omega_{p s}$ is the plasma frequency and $v_{s}$ is the velocity for each particle stream $s$. The assumption of cold streams simplifies the theoretical analysis and is justified as typically the streams under consideration have an almost Dirac delta function-like distribution function in momentum space. Complex solutions can exist for such dispersion relations below a critical wavevector $k_{c}$, the positive imaginary component of which corresponds to the linear unstable wave growth.

In the context of BOA, Albright et al. [17] have shown that the relativistic Buneman instability can potentially grow in 1D PIC simulations of a target undergoing RIT. This instability is a specific form of the general equation 1 ,

$\frac{\omega_{p i}^{2}}{\left(\omega-k v_{i}\right)^{2}}+\frac{\gamma^{-1} \omega_{p e}^{2}}{\left(\omega-k v_{e}\right)^{2}}=1$

The only streams considered in this particular scenario are a relativistic stream of electrons (subscript e) with Lorentz factor $\gamma$, and a population of ions (subscript $i$ ). The relativistic electrons are produced by ponderomotive acceleration through the volume of the target, while the expanding ion population is produced by TNSA. This instability can result in an unstable electrostatic wave growth that may drive an energy exchange between the faster moving electron stream and the slower ion stream. It is this instability that has been suggested as a potential energy exchange mechanism in BOA [17].

As previously seen from the 1D simulations, there is potential for a population of ions from the bulk material to be accelerated to velocities higher than that of the TNSA accelerated contamination layers. In such a scenario, the streaming of these two populations can lead to what is known as the ion-ion acoustic instability [22]. Again, this can be represented by a specific form of the general dispersion relation given by equation 1 ,

$\frac{\omega_{p f}^{2}}{\left(\omega-k v_{f}\right)^{2}}+\frac{\omega_{p s}^{2}}{\left(\omega-k v_{s}\right)^{2}}=1$

In this scenario only the two ion species, subscripts $f$ and $s$, are considered, representing the fast and slow ion populations, respectively. There will however also be a neutralising electron population present. It is important to note that due to the faster response time of this electron population there is only a specific region of relative drift velocities that will give rise to the ion-ion acoustic instability. This can be found to be $c_{s}<v_{d}<2 c_{s}$ by analysing the properties of the Vlasov equation for such a three component plasma [24], where $c_{s}=\sqrt{k_{B} T_{e} / m_{s}}$ is the ion acoustic speed of the slower ion population of mass $m_{s}$ with a neutralising electron temperature $T_{e}$ and $v_{d}$ is the difference between $v_{f}$ and $v_{s}$. The simulations reported herein show that as long as this criteria is satisfied the ion-ion instability growth tends to dominate the system and the thermal motion of the electron population can be neglected. Similar to the Buneman instability, this ion-ion acoustic instability leads to an energy exchange between the faster ion population and the slower.

If both RPA and RIT occur during the laser-solid interaction, it is possible for a composite scenario to arise between these two instabilities. This can be seen by incorporating the relativistic electron stream, the RPA accelerated ion stream and the TNSA ion stream together into the general dispersion given by equation 1 ,

$\frac{\omega_{p f}^{2}}{\left(\omega-k v_{f}\right)^{2}}+\frac{\omega_{p s}^{2}}{\left(\omega-k v_{s}\right)^{2}}+\frac{\gamma^{-1} \omega_{p e}^{2}}{\left(\omega-k v_{e}\right)^{2}}=1$

This can lead to an energy exchange between the three different particle populations.

By performing Fourier analysis on the longitudinal electric field component taken from the simulations, it 


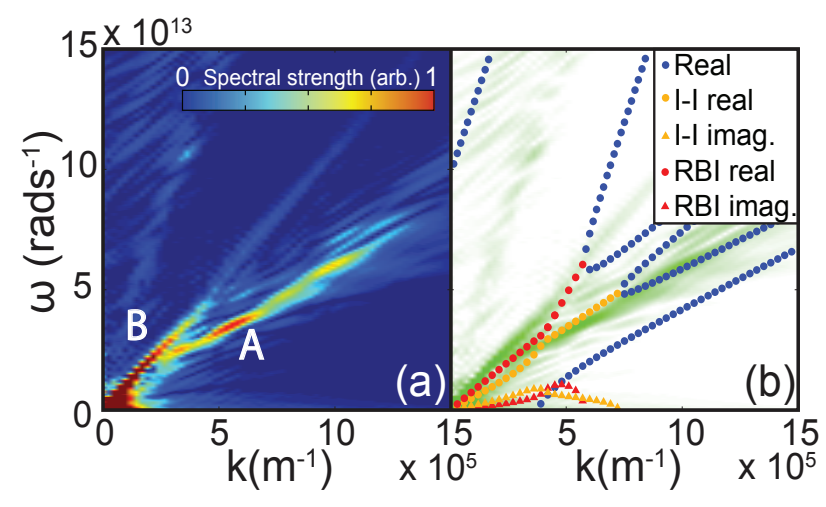

Figure 4. (a) Spectral power as a function of frequency and wavenumber for $\mathrm{t}=2.2-3.8 \mathrm{ps}$ and $\mathrm{X}=80-250 \mu \mathrm{m}$ from the $1 \mathrm{D}$ simulation. Instability excitation is observed at $\mathrm{A}$ and $\mathrm{B}$. (b) Analytic solutions to the combined dispersion relation for the ion-ion acoustic instability (I-I) and relativistic Buneman instability (RBI). The spectral power is reproduced in the background (green) to aid the comparison. The real and imaginary components are denoted with circles and triangles, respectively.

is possible to investigate the unstable wave behaviour of such instabilities in relation to the simple analytic models. In order to observe only the salient features of the electric field, the analysis is performed within a time and space window of interest. From the 1D simulations, Fig. 4(a) shows the results of performing Fourier analysis in the temporal region of 2.2-3.8 ps and spatial region of $80-250 \mu \mathrm{m}$. By taking the mean aluminium, proton and electron number densities and momenta and substituting into Eq. 4, the equation is solved to yield the approximate linear dispersion relation shown in Fig. 4(b). Whilst the equation is solved for the combined Buneman and ionion acoustic instabilities, the growing unstable waves can be attributed to the two individual instabilities. Despite the assumptions made in the linear theory, good agreement is observed when compared with the numerical dispersion relation obtained from the Fourier analysis of the 1D simulation. A clear wave can be seen to propagate at values for $\omega$ and $k$ that would be associated with the strongest growth of the ion-ion acoustic instability (feature A in Fig. 4(a)). Similarly there is a wave propagating with both a lower $\omega$ and $k$ associated with the relativistic Buneman instability (feature B in Fig. 4(a)).

Performing the same analysis on the $2 \mathrm{D}$ simulations, it is again possible to generate a numerical dispersion relation from the temporal and spatial longitudinal electric field data. This is shown in Fig. 5(a). The Fourier window was 100-400 fs in time (in order to ignore the initial shock) and 2.5-15 $\mu \mathrm{m}$ in space (avoiding the secondary instability and the expanding edges of the proton layer). In this simplified geometry, there is only one main area of unstable wave growth asso-

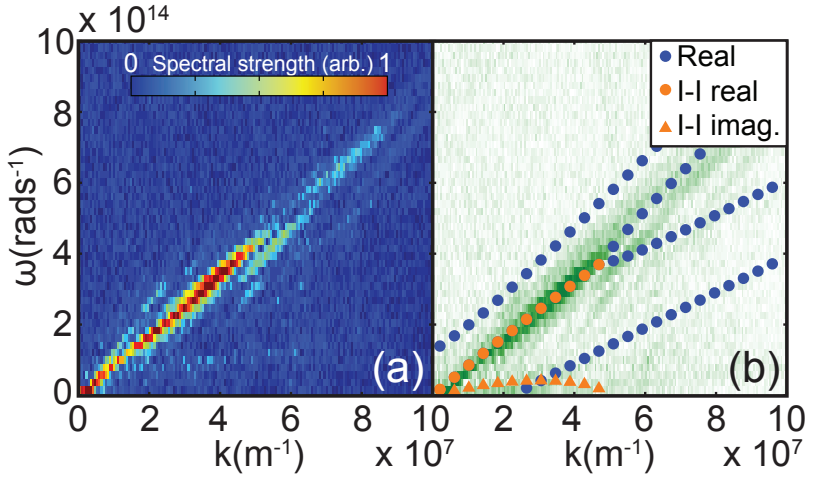

Figure 5. (a) Spectral power as a function of frequency and wavenumber for $\mathrm{t}=0.5-4.0 \mathrm{ps}$ and $\mathrm{X}=2.5-15 \mu \mathrm{m}$ from the $2 \mathrm{D}$ simulation. (b) Analytic solutions to the dispersion relation for the ion-ion acoustic instability (I-I). The spectral power is reproduced in the background (green) to aid the comparison. A clear signature of the ion-ion acoustic instability is observed.

ciated purely with the ion-ion acoustic instability. To show this, a linear analysis was again performed, using values from the initial simulation parameters, modified to compensate for the initial shock acceleration of the protons by taking the density and velocity values of the streaming populations at $0.05 \mathrm{ps}$. These values were then used to solve the dispersion relation in Eq. 3 with the results overlaid on the numerical dispersion relation as shown in Fig. 5(b). As can be seen, the dispersion properties of the growing wave from the $2 \mathrm{D}$ numerical simulation matches the maximum unstable wave behaviour predicted from the linear theory. There is additional wave behaviour seen in the numerical dispersion that corresponds to the non-linear behaviour of the instability. By varying the starting densities of the ion populations, the wave frequency, wave-vector and growth rates change accordingly with the linear theory predictions. As stated previously, if the drift velocity between the ion populations is increased beyond $2 c_{s}$ the instability does not grow, resulting in purely thermal modulations in the ion populations with no appreciable energy exchange between the two populations. For initial relative drift velocities less than $c_{s}$, the initial shock becomes the dominant process and prevents the instability growth as the fastest $\mathrm{Al}^{11+}$ ions can no longer reach the protons.

\section{Discussion}

In summary, a variety of streaming instabilities can potentially occur as the result of ultra-intense laser interactions with ultra-thin foils in which electrons and multiple ion species are accelerated. As the interaction physics changes from thermal to radiation pressure dominated, over the laser pulse duration, charged particles with a range of velocities are produced 
creating conditions for particle streaming and energy exchange. The relativistic Buneman instability has been previously identified in simulations to play a role in the BOA acceleration scheme [17]. The growth of a wave with characteristics consistent with the relativistic Buneman instability is observed in 1D simulations reported here, though there are other potential mechanisms which could give rise to this feature. In addition, energy exchange via the ion-ion acoustic instability is also observed in preexpanded multi-species targets. Whilst it is found that the accelerated energies of the bulk target ions is exaggerated in 1D simulations, there is potential for these ions to be accelerated into expanding rear surface ions particularly at higher laser intensities achievable at multi-petawatt laser facilities such as APOLLON and ELI. At higher intensities the laser radiation pressure is higher and thus the initial hole-boring drive will be enhanced. However, the increased intensity may also induce additional plasma heating and expansion, which results in an increased proton expansion velocity that may prevent the fast aluminium component from reaching the proton layer. Additional complications arise if transparency is induced earlier in a higher intensity pulse interaction. Our simplified 2D simulations (using the ion velocity and density distributions extracted from the 1D laserfoil simulations) demonstrate that the ion-ion acoustic instability can occur if the ion populations stream together. This is independent of any laser interactions or the relativistic Buneman instability. The potential therefore exists for these streaming instabilities to occur during laser-solid interactions and could lead to energy exchange between the different particle species which may in turn modify the spectral distribution of ions. Further investigation is required to determine whether there is an interaction regime in which the ion-ion acoustic instability not only occurs but results in significant energy exchange.

\section{Acknowledgements}

We acknowledge the use of the ARCHIE-WeST and ARCHER computers. This work is supported by EPSRC (grants: EP/J003832/1, EP/L001357/1, $\mathrm{EP} / \mathrm{K} 022415 / 1$ and EP/L000237/1), the US Air Force Office of Scientific Research (grant: FA865513-1-3008) and the European Unions Horizon 2020 research and innovation program (grant agreement No. 654148 Laserlab-Europe). EPOCH was developed under EPSRC grant EP/G054940/1. Data associated with research published in this paper is accessible at http://dx.doi.org/10.15129/c08cee723cca-4145-b805-ef141fee99d3.

\section{References}

[1] See H. Daido, M. Nishiuchi and A.S. Pirozhkov, Rep. Prog. Phys., 75, 056401 (2012); A. Macchi, M. Borghesi, and M. Passoni, Rev. Mod. Phys., 85, 751 (2013) and references therein

[2] S. V. Bulanov, T. ZH. Esirkepov, V. S. Khoroshkov, A. V. Kuznetsov and F. Pegoraro. Phys. Lett. A 299, 240 (2002)

[3] M. Borghesi, A. Schiavi, D. H. Campbell, M. G. Haines, O. Willi, A. J. MacKinnon, L. A. Gizzi, M. Galimberti, R. J. Clarke and H. Ruhl, Plasma Phys. Control. Fusion 43, A267 (2001)

[4] M. Roth, T. E. Cowan, M. H. Key, S. P. Hatchett, C. Brown, W. Fountain, J. Johnson, D. M. Pennington, R. A. Snavely, S. C. Wilks, K. Yasuike, H. Ruhl, F. Pegoraro, S. V. Bulanov, E. M. Campbell, M. D. Perry, and H. Powell. Phys. Rev. Lett.86, 436 (2001)

[5] S.C. Wilks, A.B. Langdon, T.E. Cowan, M. Roth, M. Singh, S. Hatchett, M.H. Key, D. Pennington, A. MacKinnon, and R.A. Snavely, Phys. Plasmas, 8, 542 (2001)

[6] J. Fuchs, P. Antici, E. d'Humières, E. Lefebvre, M. Borghesi, E. Brambrink, C.A. Cecchetti, M. Kaluza, V. Malka, M. Manclossi, and S. Meyroneinc, Nature Phys., 2, 48 (2006)

[7] L. Robson, T. Simpson, R.J. Clarke, K.W.D. Ledingham, F. Lindau, O. Lundh, T. McCanny, P. Mora, D. Neely, C.G. Wahlstrm, and M. Zepf, Nature Phys. 3, 58 (2007)

[8] P. McKenna, F. Lindau, O. Lundh, D.C. Carroll, R.J. Clarke, K.W.D. Ledingham, T. McCanny, D. Neely, A.P.L. Robinson, L. Robson, and P.T. Simpson, Plasma Phys. Control. Fusion 49, B223B231 (2007)

[9] T. Esirkepov, M. Borghesi, S.V. Bulanov, G. Mourou, and T. Tajima, Phys. Rev. Lett. 92, 175003 (2004)

[10] A. Macchi, S. Veghini, T.V. Liseykina, and F. Pegoraro, New. J. Phys. 12, 045013 (2010)

[11] S. Kar, K.F. Kakolee, B. Qiao, A. Macchi, M. Cerchez, D. Doria, M. Geissler, P. McKenna, D. Neely, J. Osterholz, R. Prasad, K. Quinn, B. Ramakrishna, G. Sarri, O. Willi, X. Y. Yuan, M. Zepf, and M. Borghesi, Phys. Rev. Lett. 109, $185006(2012)$

[12] V.A. Vshivkov, N.M. Naumova, F. Pegoraro, and S.V. Bulanov, Phys. Plasmas, 5, 2727 (1998)

[13] R.J. Gray, D.A. MacLellan, B. Gonzalez-Izquierdo, H.W. Powell, D.C. Carroll, C.D. Murphy, L.C. Stockhausen, D.R. Rusby, G.G. Scott, R. Wilson, N. Booth, D.R. Symes, S.J. Hawkes, R. Torres, M. Borghesi, D. Neely and P. McKenna, New J. Phys., 16, 093027 (2014)

[14] B. Gonzalez-Izquierdo, R.J. Gray, M. King, R.J. Dance, R. Wilson, J. McCreadie, N.M.H. Butler, R. Capdessus, S. Hawkes, J.S. Green, M. Borghesi, D. Neely and P. McKenna, Nature Phys., At Press (2015)

[15] H.W. Powell, M. King, R.J. Gray, D.A. MacLellan, B. Gonzalez-Izquierdo, L.C. Stockhausen, G. Hicks, N.P. Dover, D.R. Rusby, D.C. Carroll, H. Padda, R. Torres, S. Kar, R.J. Clarke, I.O. Musgrave, Z. Najmudin, M. Borghesi, D. Neely and P. McKenna, New J. Phys., 17, 103033 (2015)

[16] O. Buneman, Phys. Rev. 115, 503 (1959)

[17] B.J. Albright, L. Yin, K.J. Bowers, B.M. Hegelich, K.A. Flippo, T.J.T. Kwan, and J.C. Fernández, Phys. Plasmas 14, 094502 (2007)

[18] L. Yin, B.J. Albright, B.M. Hegelich, and J.C. Fernández, Laser Part. Beams 24, 291 (2006)

[19] D. Jung, B.J. Albright, L. Yin, D.C. Gautier, R. Shah, S. Palaniyappan, S. Letzring, B. Dromey, H.C. Wu, T. Shimada, R.P. Johnson, M. Roth, J.C. Fernández, D. Habs and B.M. Hegelich, New. J. Phys. 15, 123035 (2013)

[20] S.V. Bulanov, D.V. Dylov, T.Z. Esirkepov, F.F. Kamenets, 
and D.V. Sokolov, Plasma Phys. Reports 31369 (2005)

[21] T. Nakamura, S.V. Bulanov, T.Z. Esirkepov, and M. Kando, Phys. Rev. Lett., 105135002 (2010)

[22] S.P. Gary and N. Omidi, J. Plasma Phys. 37, 1, 45-61 (1987)

[23] T.J.M. Boyd and J.J. Sanderson, The physics of plasmas, Cambridge University Press, (2003)

[24] T. Fujita, T. Ohnuma and S. Adachi, Plasma Phys. 19, 9, 875 (1977)

[25] T.D. Arber, K. Bennett, C.S. Brady, A. Lawrence-Douglas, M.G. Ramsay, N.J. Sircombe, P. Gillies, R.G. Evans, H. Schmitz, A.R. Bell, and C.P. Ridgers, Plasma Phys. Control. Fusion 57, 113001 (2015)

[26] F. Fiuza, A. Stockem, E. Boella, R.A. Fonseca, L.O. Silva, D. Haberberger, S. Tochitsky, C. Gong, W.B. Mori, and C. Joshi, Phys. Rev. Lett. 109, 215001 (2012)

[27] F. Fiuza, R.A. Fonseca, J. Tonge, W.B. Mori, and L.O. Silva, Phys. Rev. Lett. 108, 235004 (2012) 